\title{
Automated Analysis of Repetitive Joint Motion
}

\author{
ChunMei Lu and Nicola J. Ferrier, Member, IEEE
}

\begin{abstract}
Automated measurement, analysis, and comparison of human motion during performance of workplace tasks or exercise therapy are core competencies required to realize many telemedicine applications. Ergonomic studies and telemonitoring of patients performing rehabilitation exercises are examples of applications that would benefit from a representation of complex human motion in a form amenable to comparison. We present a representation of joint motion suitable for the analysis of multidimensional angular joint motion time series data. Complex motion is reduced to a concatenation motion segments, where simple dynamic models approximate the observed motion on each segment. This compact representation still enables measurement of statistics familiar to ergonomics practitioners such as cycle length and task duration. An algorithm to obtain this representation from observed motion data (time series) is given. We introduce a metric, based on a kinetic energy-like measure, to compare motions. Experiments are presented to demonstrate the representation, its relationship to previous measures and the applicability of the kinetic energy metric for motion comparison.
\end{abstract}

Index Terms-Joint motion analysis, telemonitoring, ergonomics.

\section{INTRODUCTION}

Q UANTIFICATION of the relationship between posture, repetitiveness, and risk of injury while performing a workplace task requires measurement and analysis of human motion during task execution. Telemonitoring of patients may also utilize measurement and comparison of human motions. However, complex geometry in motion (e.g., full body motion) is rarely analyzed and compared-reasons may include the difficulty in acquiring data, the lack of computational or analytic tools for analyzing the data, and the lack of metrics to compare motions between different subjects. In most ergonomic studies large quantities of data must be analyzed for reliable determination of exposure response relationships, assessing risk of injury, or detecting performance degradation.

Work-related cumulative trauma disorders (CTDs) are now recognized as a major occupational health problem. Disorders such as carpal tunnel syndrome, tenosynovitis, tendinitis, and chronic muscle strain have been linked to the jobs that are repetitive, require high forces, and require continuous or repeated extreme or awkward postures [4]-[6]. Significant effort has been made to quantify the actual relationship between posture, force,

Manuscript received November 19, 2001; revised October 8, 2002 and June 9, 2003. This work was supported by NSF (IRI-9703352) and by a University of Michigan Center for Occupational Health and Safety Engineering Pilot Research Award.

C. M. Lu is with the Department of Mechanical Engineering and Center for Mathematical Sciences, University of Wisconsin-Madison, WI 53706 USA.

N. J. Ferrier is with the Departments of Mechanical Engineering and Biomedical Engineering, University of Wisconsin-Madison, WI 53706 USA

Digital Object Identifier 10.1109/TITB.2003.821309 repetitiveness and risk of injury. Silverstein et al. [23] observed an increase in the risk of carpal tunnel syndrome and tendinitis when performing jobs with a fundamental work cycle of less than $30 \mathrm{~s}$ that were performed for more than 50 percent of the time. Armstrong et al. [3] obtained posture data for the hand and wrist using traditional industrial engineering elemental analysis methods based on observing cinematographic records for estimating the frequencies of various hand positions used for sewing. Priehl [20] developed the "posturegram" method that provided information on the locations and movements of body parts in three coordinates planes. Karhu et al. [19] used a method in which specific postures were assigned ordinal values. Another posture classification method called posture targeting was used by Corlett et al. [8] which had continuous posture resolution in the vertical and horizontal planes with postures recorded at variable intervals using time sampling. Descriptive statistics, such as total time and average time spent in each posture have been used for reducing posture data (Keyserling [11]; Wells [24]). Marras and Schoenmarklin [16] measured wrist flexion in terms of angular motion (both velocity and acceleration). Statistical analysis of these measures revealed that velocity and acceleration data of wrist motion discriminated significant differences between low- and high-risk groups. Spectral analysis was adopted by Radwin et al. [21] as an analytical method for characterizing repetitive wrist motion and postural stress. The method was later tested by Yen [25] and compared against conventional observational posture analysis on a variety of industrial jobs in the field. It was concluded that spectral analysis was a potentially efficient method for quantifying exposure to physical stress in repetitive manual work.

All of the aforementioned ergonomic studies are either based on human observational assessment or only a "lumped" or averaged value is used to describe motion (such as frequency, duration, etc.). Moreover most of the above studies only consider wrist motions because there is no "standard" to measure, represent and compare the motions with more than one degree of freedom (e.g., whole body or upper body). Additionally, raw time series data is difficult to compare as small variations in the time scale are difficult to accommodate in standard metrics. To date, there are no quantitative guidelines or standards available for determining the level of acceptable exposure to motion stresses in order to avoid reduced work performance and prevent injuries. Most epidemiological studies have only quantified the exposure factors in term of numerical rating levels (usually from 1 to 10) assigned by the observational human analyst which is subjective, lacks resolution, and is time consuming.

A more efficient, repeatable, and quantifiable method for the analysis of human motion is desirable. The ability to compactly represent human motion affords the ability to implement 
systems for telemonitoring, telediagnostics, and teleconsultation. In ergonomics, a compact representation of motion will enable collection and transmission of data for establishing exposure-response relationships, and, once guidelines have been established, the same representation would be useful for development of monitoring systems. In rehabilitation, remote or in-home monitoring of patient exercises would reduce patient visits to therapists while still ensuring the patient maintains correct form, ensuring patient compliance with exercise regimes, and monitoring patient progress.

In this paper, we present a representation of multidimensional joint motion data that decomposes a repetitive motion into "motion segments" and on each segment a parameterized dynamic model is fit. The model parameters are insensitive to small time variations in the motion duration hence the set of model parameters form a time-invariant representation of each segment of the motion. Based on the model parameters, we present a "kinetic energy-like" measure that can be used to compare task motions between different subjects and different task conditions. Our dynamic model representation of motion is consistent with previous approaches in that motion statistics (frequency, amplitude, cycle length) can be obtained.

In the remainder of this paper we present the details of our analysis tools for joint angle times series motion data. We present methods to: 1) segment the data into "primitive" elements, 2) represent motion data on each segment using parameters of a dynamic model fit to the motion, and 3) compare motions based on a "kinetic energy-like" measure. Our representation extends the practitioners usual metrics to enable measurement of complex multijoint motion. Experimental results are presented to demonstrate the ability to extract, measure and compare subject motion.

\section{COMPLEX Human Motion Data}

\section{A. Motion Data Acquisition}

Numerous applications require the ability to obtain human movement geometry and many sensing techniques have been used to acquire human motion [9], [17]. Existing technologies for measuring human motion often use specialized hardware. For example, electromagnetic tracking devices function by measuring the strength of the magnetic fields generated by transmitters worn by subjects. Ergonomic assessment often uses electrogoniometers, mechanical tracking devices constructed to measure details of geometry. Video is a promising technology and research systems have been developed to measure human motion using digital video data [14].

Most of the motion acquisition systems either directly or indirectly obtain joint angles recorded as a time indexed motion sequence. While this data can be used directly by practitioners, alternate representations of the motion data may be more compact, easier to analyze and to compare. We next consider methods to fit dynamic models to the data to allow classification and compact representation of the time series motion data.

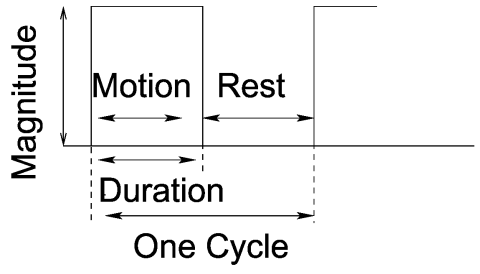

Fig. 1. The Hand Activity Level is often used for repetitive motion assessment.

\section{B. Dynamic Model Representation}

The raw joint motion data is indexed by time (or, equivalently, frame number for data acquired from video). In this form it is difficult to compare motions and analyze tasks. Extracting dynamic parameters from the raw data permits a compact representation of the data. Numerous representations are possible.

One motion metric in use by ergonomists is the Hand Activity Level (HAL) [1]. Each task is described on a scale of 1-10 in terms of the motion of the hands (and the forces applied by the hands). Motion is determined based on frequency, duration and magnitude of the motion (Fig. 1). The frequency or cycle times measure how often a motion is performed, the duration expresses the percentage of the cycle length during which the hand is moving. The amplitude or magnitude measures the "size" of the motion, typically the angular displacement of the wrist [1]. For a single joint, these quantities can be determined by fitting a damped harmonic motion model to the joint angle time series data. Thus descriptive statistics, such as average and peak values in time and frequency domain have been used to represent motion data. These statistics only make sense in one-dimensional (1-D) data, i.e., motion of a single joint. Extensions to higher dimensions have not been found in the literature.

In [15], we presented an algorithm to automatically segment multidimensional data into elemental segments and used system identification techniques to fit dynamical models, such as the autoregressive (AR) model, to the segmented data. AR models relate the current state $x(t)$ to one or more previous values $x(t-$ $1), x(t-2), \ldots$ of the same series. In a first-order AR model

$$
x(t)=c_{1} x(t-1)+e(t)
$$

the state $x(t)$ depends partly on the previous state $x(t-1)$ and partly on a random term (noise) $e(t)$. The AR coefficient $\left(c_{1}\right.$ in this example) must lie between -1 and +1 and it measures the degree of correlation between successive values of the series. The second-order AR model supposes that the state $x(t)$ is a linear aggregate of the two previous states and of a random term

$$
x(t)=c_{1} x(t-1)+c_{2} x(t-2)+e(t) .
$$

For upper extremity motion (torso and arms), a second-order AR model was used because the first-order dynamical model can not adequately describe motions of interest, such as motion of human arms and hands, as pointed out in [7]. In this formulation the state vector at time $t_{k}, X\left(t_{k}\right)$, is composed of the joint angles of interest. For the upper extremities, we will use

$$
X\left(t_{k}\right)=\left(\alpha_{w}\left(t_{k}\right), \alpha_{e}\left(t_{k}\right), \alpha_{s}\left(t_{k}\right)\right)^{T}
$$


where $\alpha_{w}, \alpha_{e}$, and $\alpha_{s}$ are the wrist, elbow and shoulder angles, respectively, at time $t_{k}$. The second-order AR process has the form

$$
\begin{aligned}
X\left(t_{k}\right)-\bar{X}=A_{2}\left(X\left(t_{k-2}\right)\right. & -\bar{X}) \\
& +A_{1}\left(X\left(t_{k-1}\right)-\bar{X}\right)+B_{0} e_{k}
\end{aligned}
$$

where the matrices $A_{2}, A_{1}$, and $B_{0}$ encode the system dynamics, $e_{k}$ is Gaussian noise with zero mean and variance $\sigma_{k}^{2}$, and $\bar{X}$ is the mean of $X$. Equation (2) can be expressed more compactly in matrix form

$$
\mathcal{X}\left(t_{k}\right)-\overline{\mathcal{X}}=A\left(\mathcal{X}\left(t_{k-1}\right)-\overline{\mathcal{X}}\right)+B e_{k}
$$

where

and

$$
\mathcal{X}\left(t_{k}\right)=\left(\begin{array}{c}
X\left(t_{k-1}\right) \\
X\left(t_{k}\right)
\end{array}\right), \quad \overline{\mathcal{X}}=\left(\begin{array}{c}
\bar{X} \\
\bar{X}
\end{array}\right), B=\left(\begin{array}{c}
0 \\
B_{0}
\end{array}\right)
$$

$$
A=\left(\begin{array}{cc}
0 & I \\
A_{2} & A_{1}
\end{array}\right) \text {. }
$$

The system matrix of the process $A$ fully describes the deterministic part of the motion model, and matrix $B$ describes the stochastic part of the model.

We define primitive segments as motions that can be described by this simple dynamic model. A task motion is segmented into these segments by using our technique [15] which is briefly reviewed here.

\section{Recursive Least Square Segmentation Algorithm}

Given a set of measurements $X\left(t_{k}\right)$ and a dynamic model, such as the AR process above (3), the dynamic model fitting problem is one of determining the parameters, i.e., the elements in the matrix $A$. The motion segmentation problem is one of detecting the times at which the dynamical parameters change. We formulate the motion segmentation problem using recursive parameter estimation (and by the term recursive we mean that the new value of the estimated parameter is equal to the previous value plus a correcting term which will depend on the most recent measurements). Our segmentation method is an extension of Andersson's segmentation for 1-D systems.

A typical description of a time-discrete (1-D) system with varying parameters is the state space model

$$
\left.\begin{array}{c}
\theta(t+1)=\theta(t)+w(t) \\
y(t)=\varphi^{T}(t) \theta(t)+e(t)
\end{array}\right\}
$$

where $\theta(t)$ is an $n$-dimensional vector containing the true parameters describing the system at time $t, \varphi(t)$ is a vector containing the old inputs and outputs (i.e., $x(t-1), x(t-2), \ldots$ and $y(t-1), y(t-2), \ldots)$, and $e(t)$ and $w(t)$ are disturbances with variances $R_{2}(t)$ and $R_{1}(t)$, respectively. The well known Kalman filter provides a possible estimation for constant $\theta$. However, for systems where the parameters describing the system are not constant Andersson [2] presented a segmenta- tion approach based on finite-Gaussian sum approximation. A problem common to both of the algorithms is that they require some priori knowledge of the system to be identified, i.e., $R_{1}(t)$ and $R_{2}(t)$ (the variance of the noise). Without this information, over- or undersegmentation will occur. Estimation of parameters $R_{1}$ or $R_{2}$ is difficult and, hence, generally these values are attained by trial and error. Considering the high dimension case of interest here, in which we need to monitor the parameters in two $n \times n$ matrices $\left(A_{1}\right.$ and $A_{2}$ ), was impossible to test and try all the possibilities. We present a more robust and efficient high-dimensional segmentation method.

Using $W(t)$ to model the changing parameters, the AR model we considered above (3) can be put into the form of the state space model (6)

$$
\left.\begin{array}{c}
A(t+1)=A(t)+W(t) \\
Y(t)=A(t) Y(t-1)+E(t)
\end{array}\right\}
$$

where

$$
Y(t)=\mathcal{X}\left(\mathrm{t}_{\mathrm{k}}\right)-\overline{\mathcal{X}} \quad \text { and } \quad A=\left(\begin{array}{cc}
0 & I \\
A_{2} & A_{1}
\end{array}\right) .
$$

Minimizing the criterion function

$$
\begin{aligned}
V_{t}(\mathcal{A}) & =\sum_{k=1}^{t} \beta(t, k)\|Y(k)-A Y(k-1)\|^{2} \\
\text { with } \beta(t, k) & =\prod_{j=k+1}^{t} \lambda(j)
\end{aligned}
$$

we obtained the following high-dimensional recursive least square (HRLS) algorithm

$$
\begin{aligned}
\hat{A}(t)= & \hat{A}(t-1)+L(t)(Y(t)-\hat{A}(t-1) Y(t-1))^{T} \\
L(t)= & \frac{P(t-1) Y(t-1)}{\lambda(t)+Y^{T}(t-1) P(t-1) Y(t-1)} \\
P(t)= & \frac{1}{\lambda(t)}\{P(t-1) \\
& \left.-\frac{P(t-1) Y(t-1) Y^{T}(t-1) P(t-1)}{\lambda(t)+Y^{T}(t-1) P(t-1) Y(t-1)}\right\}
\end{aligned}
$$

where $P(t)$ is adaptation gain, and $\lambda(t)$ is called the forgetting factor, which discounts old measurements ( $\lambda$ was chosen as a constant 0.98 in our experiments).

Our multidimensional motion segmentation algorithm combines the above HRLS algorithm with a recursive least square (RLS) algorithm. First, HRLS was applied to the input data to estimate the AR model, giving a prediction of motion parameter matrix, $A(\mathrm{t})$. The changes of our multivariable system were represented by the difference matrix of adjacent parameter matrices $A(\mathrm{t})-A(\mathrm{t}-1)$. Second, the MSE (mean square error) of the difference matrix was computed and an additional 1-D RLS procedure was applied to this 1-D system to detect the segmentation points (places where the parameter matrix changes significantly). 

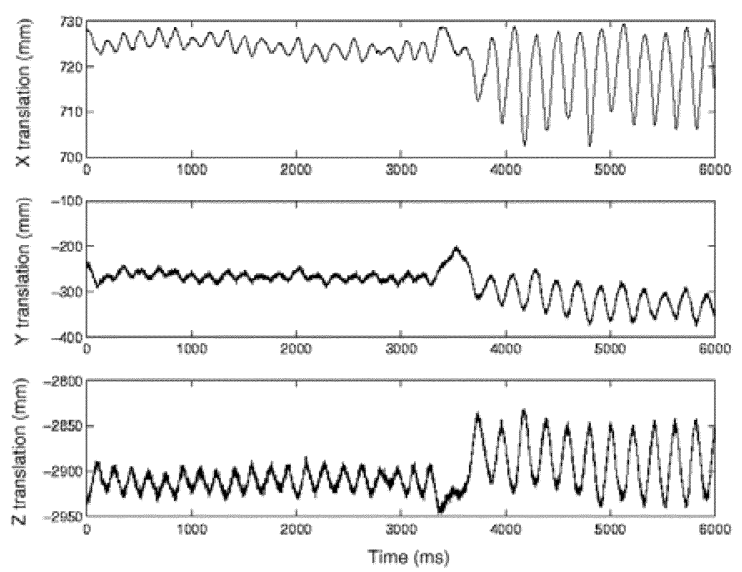

(a)

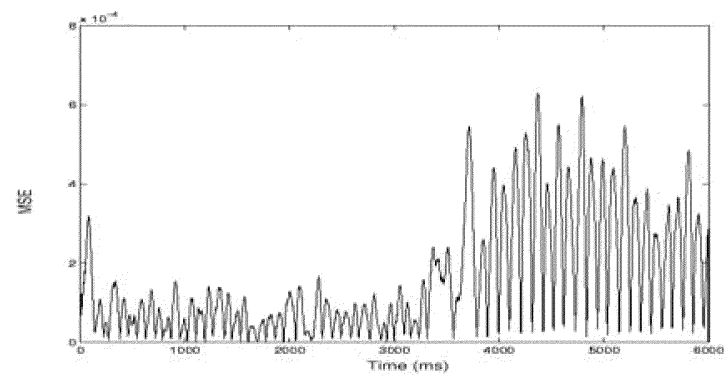

(c)
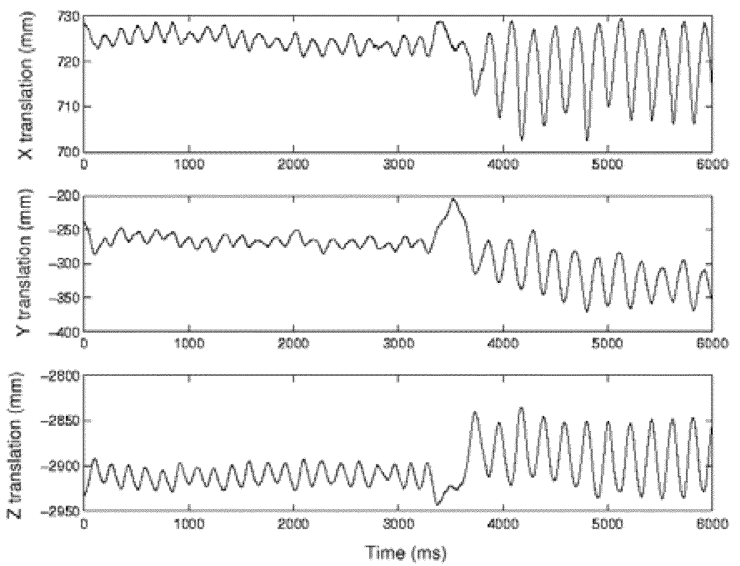

(b)

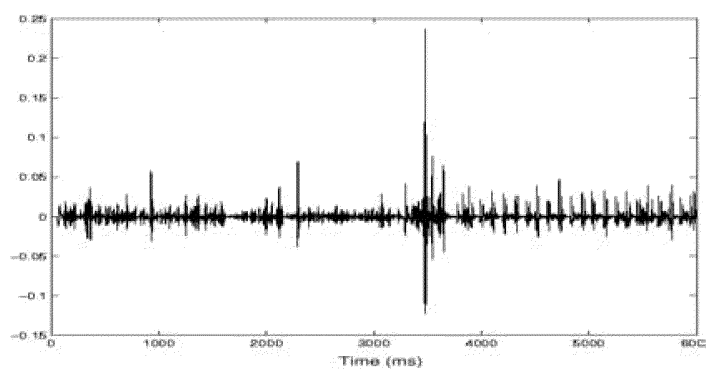

(d)

Fig. 2. The steps for multidimensional segmentation: (a) Original motion data of a human turning crank. The motion state vector is comprised of the three translation components. The plots from top to bottom are X-translation, Y-translation, and Z-translation, respectively. (b) Low-pass filtered motion data. (c) MSE of difference matrix $a(t)=\|\hat{A}(t)-\hat{A}(t-1)\|$ for the estimated dynamic parameters. (d) A high-pass filter of the MSE. Peak points in this graph are used to determine the segmentation locations- - here the segmentation at time corresponding approximately to frame number 3500 breaks the motion in (a) into two segments.

Unlike the previous system-identification-based segmentation methods, which make the segmentation completely based on the output of the system identification algorithms, we determined the segmentation step by step according to the model-fitting error. By doing this, we avoided having the performance of the algorithm depend on how much prior knowledge we had about the system.

To summarize, the multidimensional segmentation algorithm is given as follows: (Fig. 2 shows the result of applying the algorithm to hand motion measured while a subject was turning a crank, first using a handle near the axle, then changing to use a handle further from the axle).

Step 1) The motion data was filtered to reduce noise. A low-pass filter was applied to smooth the data and the mean value of the data was then subtracted to obtain $Y(t)(8)$.

Step 2) HRLS was applied to the motion data (9). The the difference matrix, $\hat{A}(t)-\hat{A}(t-1)$ was computed for adjacent parameter matrices. This difference matrix gave a reasonable measure of change in the motion parameters.

Step 3) The norm of this difference matrix was computed at each time unit, converting the multivariable system to a 1-D time series $a(t)=\|\hat{A}(t)-\hat{A}(t-1)\|$. Several kinds of matrix norms were tried and we found their performance to be similar with respect to the segmentation achieved. The widely used MSE was adopted in our experiments. For $A=\left(a_{i j}\right)_{m \times n}$, this norm is defined as

$$
\|A\|=\frac{1}{m n} \sum_{i=1}^{m} \sum_{j=1}^{n}\left|a_{i j}\right|^{2} .
$$

Note: The elements of the dynamic system matrix $A(t)$ will have elements of different units (e.g., in the second-order harmonic oscillator the elements will include the damping coefficient and the natural frequency). The error, MSE, between adjacent matrices calculated as given above represents a distance in some parameter space. The units for this norm will depend on the particular state vector and dynamic system model. Henceforth units will be omitted in all MSE plots.

Step 4) A 1-D RLS (6) was applied to the MSE of the difference matrix, i.e., $a(t)=c_{1} a(t-1)+e(t)$.The parameter of this time series, $c_{1}$ was estimated.

Step 5) Peak points of the resulting 1-D system $a(t)$ from step 4 were determined and used as locations of segmentation points. When MSE is large, then the estimated system dynamic parameters $\hat{A}(t)$ and $\hat{A}(t-1)$ differ. A high-pass filter was applied to determine these points. 


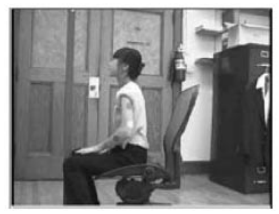

(a)

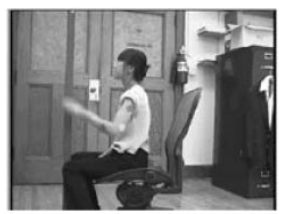

$(\mathrm{ac}$

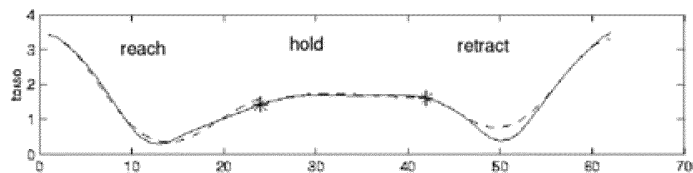

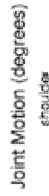
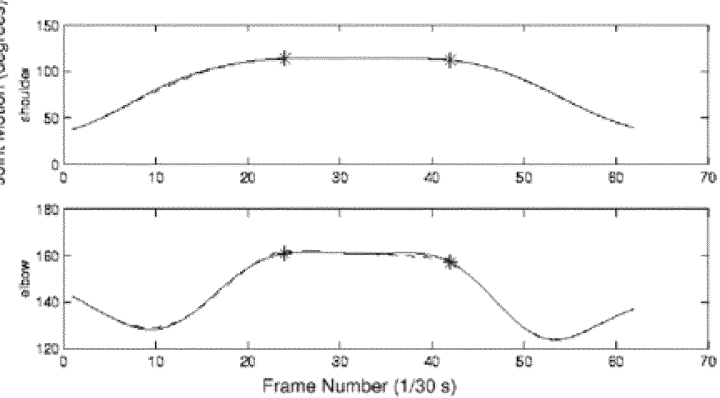

(e)

Fig. 3. (a)-(d) Four images from the motion sequences of a human subject performing a reaching task. (e) One cycle of motion from the reaching sequence with the segmentation and fitting result. In this motion, three segments were found and the computed segmentation points are marked with asterisks $*$. Original motion data is shown with a solid line, the harmonic model fit to the data is shown with a dashed line.

Step 6) Dynamic model fitting was performed on each segmented interval, fitting the second-order AR model (3). If the fitting error was greater than a predetermined threshold, the algorithm returned to step 5 and used additional peaks to obtain a finer segmentation. This was repeated until the fitting threshold was satisfied. Although not used in the work presented here, a merge technique [12] could be adopted to combine data from adjacent segments that agree on the system parameters. This could be useful to avoid oversegmentation when using extremely noisy data.

Fig. 3 gives an example of the motion segmentation algorithm applied to motion data obtained from a person performing a simple task of reaching targets at different heights. The motion data is acquired by our vision based motion measurement system [14], Fig. 4 shows the original motion time series data. The torso, shoulder, and elbow angles were measured (relative to a "zero" position), all in degrees. Four example images from the motion sequences used to generate the data are shown in Fig. 3(a)-(d). Motion data was segmented by the multisegmentation algorithm presented above. Example segmentation and fitting results are illustrated in Fig.3(e). For each cycle (reach target, pause, retract hand to resting position), it was found three
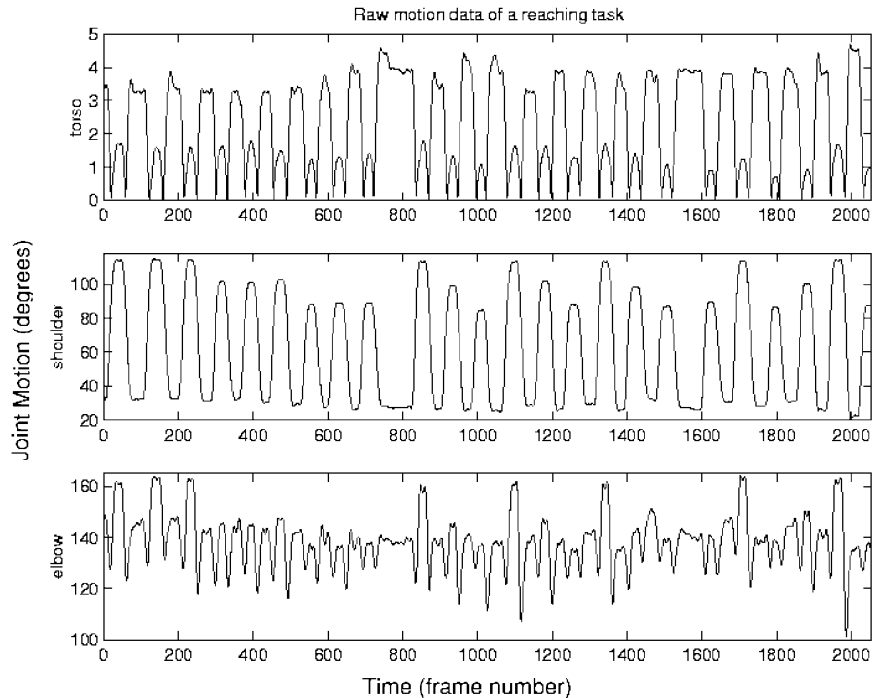

Fig. 4. Raw motion data for many cycles of the reaching task. Data is given for three limbs: torso, shoulder, and elbow, all in degrees. In the second row of data (the elbow), one can easily detect the three different targets based on the magnitude of the motion.

segments best described this motion. In the figure, segmentation points are marked by asterisks. These segments can be roughly described as a reach toward the target, a short hold at the target and a return movement from the target. Original motion data is shown with a solid line, the fitted model is plotted with a dashed line.

The algorithm segments multidimensional data into a series of elemental segments such that a simple dynamical system describes the motion on each segment. Using this algorithm, a motion can be represented as a sequence of primitive motions $\left\{\left(A_{i}, B_{i}, N_{i}\right), i \in 1, \ldots, P\right\}$ where $A_{i}, B_{i}$ represent the dynamics of the $i$ th motion segment, $N_{i}$ is the number of frames for that segment and $P$ is the number of segments in the motion. On each segment, we can extract natural frequencies using spectral analysis or the Yule-Walker AR method (see [12] for details). Pattern recognition techniques can be applied to detect specific motions and events [13]. In the following section, the dynamic parameters are used to fit a harmonic motion model to each segment which enabled the use of descriptive statistics for motion analysis.

\section{Damped Harmonic Motion Parameters}

Previous human motion studies have performed elementary analysis to analyze and reduce the motion data whereby complex tasks are broken down into "elements" in each work cycle by a human analyst. Descriptive statistics, such as average and peak values in time and frequency domain have been used to represent the data in each element. These statistics alone are limited in their ability to fully characterize the motion data. Instead of breaking motion data to pieces by hand, our segmentation algorithm performs this task automatically. Our algorithm segmented motion data into elements such that a single dynamic model could be fit on each "element" of the motion data. The dynamic model parameters provide a more compact representation of the motion. In the example given in the previous section 
(reaching), each segment corresponded to elements that may be used by a human observer (e.g., reach, pause, retract), however, for many motions the segments are not typically in one-to-one correspondence with elements used by human observers. The dynamic model fitting is based on the ability to describe a motion with a simple model, not on "higher level" descriptions of a task. For example, in analyzing a kick-boxer performing a series of moves, a human observer recognized six moves, however, the dynamic model description required ten segments.

To use descriptive statistics, after segmentation we consider each joint independently. Recall $X\left(t_{k}\right)=\left[x_{1}\left(t_{k}\right), \ldots, x_{n}\left(t_{k}\right)\right]$ denotes the $n$-dimensional state vector at time $t_{k}$ where each $x_{i}\left(t_{k}\right), 1 \leq i \leq n$ is a discrete 1-D time series signal (e.g., joint angle data from $n$ joints). Because the multidimensional segmentation algorithm provides a consistent segmentation on all $x_{i}\left(t_{k}\right), 1 \leq i \leq n$, we applied a 1-D fitting routine from [7] on each $x_{i j}\left(t_{k}\right)$, (the $i$ th component of the motion data on the $j$ th segment). This fitting estimated the fitting parameters $A_{i j}$, where $s$ is the number of segments and $A_{i j}$ is $2 \times 2$ matrix, $1 \leq i \leq n, 1 \leq j \leq s$.

The second-order AR process is a special class of Gaussian Markov processes of order 2. It is known that a Markov process in continuous time is governed by a stochastic differential equation [10], called the Fokker-Plank or Kolmogorov equation. The sample path of such a process in continuous time in 1-D is a damped harmonic oscillation

$$
x(t)=\alpha \exp (-\beta t) \cos (2 \pi f t+\phi)+T
$$

where $\alpha, \beta, f, \phi$, and $T$ represent, respectively, the amplitude, damping rate, frequency, phase, and offset information of the damped harmonic oscillation motion. For the AR process, the different harmonic modes are determined by the eigenvalues, $\lambda_{m}$, of the fitting parameter matrix $A$. The damping rate $\beta_{m}$ and frequency $f_{m}$ of the eigenvalues $\lambda_{m}$, given the sampling frequency $\tau$, are

$$
\beta_{m}=\frac{1}{\tau} \log \frac{1}{\left|\lambda_{m}\right|} \quad \text { and } \quad f_{m}=\frac{1}{2 \pi \tau} \arg \lambda_{m} .
$$

Given the damping rate and frequency information from eigenvalues of $A$, amplitude, phase, and offset information can be obtained by minimizing the error between harmonic model (10) and the original motion data.

When estimated from 1-D data, $A_{i j}$ has two eigenvalues $\lambda_{1}$ and $\lambda_{2}$ which correspond to one mode if both eigenvalues are complex and two modes otherwise. Harmonic modes with a high damping rates influence the motion only for a very short time, while the least damped modes characterize the motion. Thus, we considered the first mode of the motion in the case of two modes. The phase information can be determined from the amplitude in a damped harmonic motion model and thus is ignored from the motion representation. Therefore the 4-D vector $\left[\alpha_{i j}, \beta_{i j}, f_{i j}, T_{i j}\right]$ was used as a compact representation of the motion data $x_{i j}\left(t_{k}\right)$ because these four terms were sufficient to determine a damped harmonic motion that could be fitted to the data $x_{i j}\left(t_{k}\right), 1 \leq i \leq n, 1 \leq j \leq s$.
TABLE I

STATISTICS OF THE MOTION OF THREE JoINTS OF ONE SUBJECT REACHING A TARgET From A LOW CHAIR (FIRST THREE Rows) AND A HigH CHAIR (LOWER Three Rows). THE Motion IN EACH SEGMENT Is MOdELED AS AN EXPONENTIALLY DECAYING HARMONIC MOTION

\begin{tabular}{c|cccc|ccc}
\hline $\begin{array}{c}\text { Seg- } \\
\text { ment }\end{array}$ & $\begin{array}{c}\text { Ave. } \\
\text { Len. } \\
\text { (frames) }\end{array}$ & $\begin{array}{c}\text { Decay } \\
\text { Const } \\
(\mathrm{s})\end{array}$ & $\begin{array}{c}\text { freq. } \\
(\mathrm{Hz})\end{array}$ & Period & Trames) & Amplitude \\
\hline Low & 15 & -0.32 & 0.76 & 39.45 & 1.119 & 0.915 & 0.369 \\
1 & 29 & -0.14 & 1.13 & 26.58 & 0.012 & 0.027 & 0.996 \\
2 & 18 & 0.34 & 0.95 & 31.43 & 1.276 & 0.402 & 0.571 \\
3 & 20 & -2.60 & 0.68 & 44.17 & 0.975 & 0.629 & 0.398 \\
\hline High & 22 & -0.19 & 0.87 & 34.58 & 0.065 & 0.416 & 0.933 \\
1 & 20 & 2.05 & 0.58 & 51.73 & 0.940 & 0.240 & 0.576 \\
2 & &
\end{tabular}

Fig. 5 graphically shows the four parameter values on each segment of a motion (and the numeric values are given in Table I) for the reaching task described earlier. The motion (reaching a target) was repeated for 10 cycles and each cycle was segmented into three components. The beginning of each motion cycle is indicated by ".." The feature value on each motion cycle is represented as a step function which changes step at each segment. In our experiments, for each cycle we found a repeating pattern of dynamic parameter values and in other work we show that these parameters can be used in cluster analysis techniques for detection of motion events [13]. Next, we show how these parameters were used to compare motions.

\section{E. A Kinetic Energy Measure}

The dynamic motion parameters describe the motion, however, comparison between motions requires a metric. Spectral analysis has been suggested as a convenient method for directly computing repetition and average information for the joint angle data, using a metric called root mean square (rms) [21]. However, frequency information is lost in the metric. Kinetic energy, when computed in the frequency domain, gives an expression that combines both the magnitude and frequency information and hence can also be used as a metric of repetitiveness. It will be demonstrated that the kinetic energy metric achieves the goal of a frequency-weighted filter (suggested as desirable in [22]) and combines both the rms and frequency information.

Our notation and terminology follow from [21], [22]. In their work, the term element is defined as the set of movements contained between two arbitrary, but distinct break points associated with a task (we have previously used the term segment for the break points determined by our algorithm, which do not necessarily correspond to the elements determined by human observers). A cycle is a series of motions that are performed repeatedly. When a series of movements that are performed repeatedly are part of a cycle, they are called subcycles. Sometime subcycles can be combined into longer subcycles. When a series of repetitive movements, or subcycles, are combined until they can not be combined any further, it is called a fundamental cycle.

For comparison, we briefly summarize the methods given in [21], [22]. Sampled posture data were divided into segments $L$ points in length, corresponding to individual elements. In spectral analysis, for each element $n$ in its $m$ th cycle, the posture data 

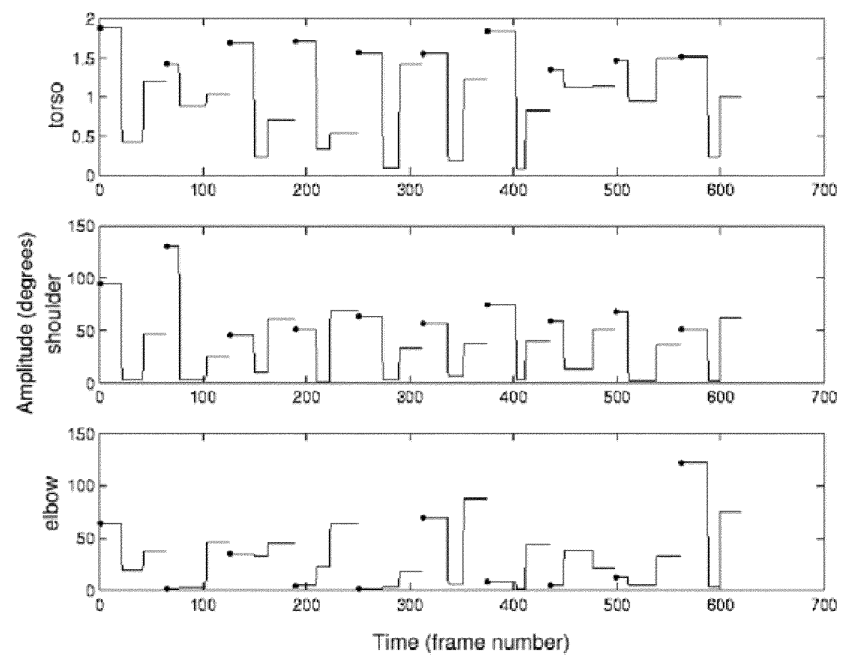

(a)
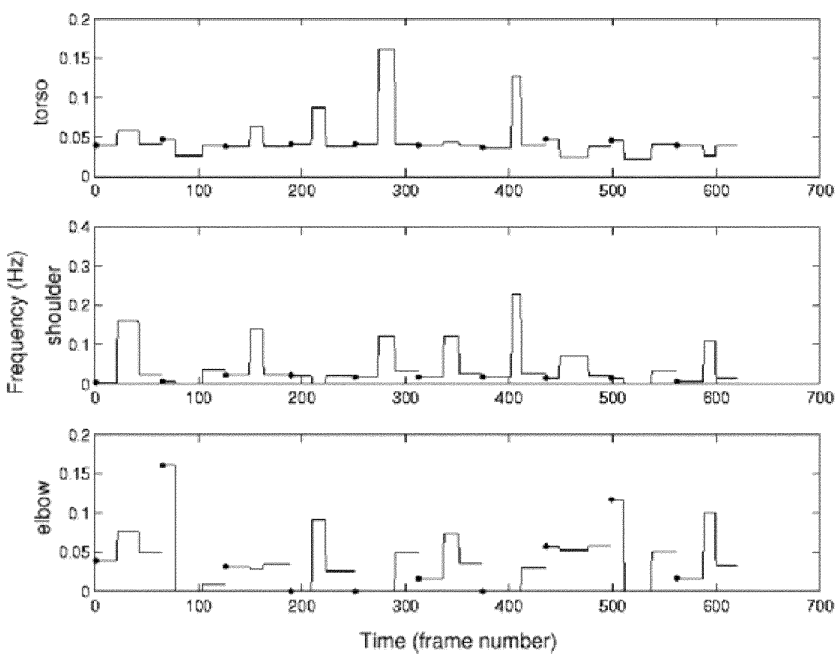

(c)
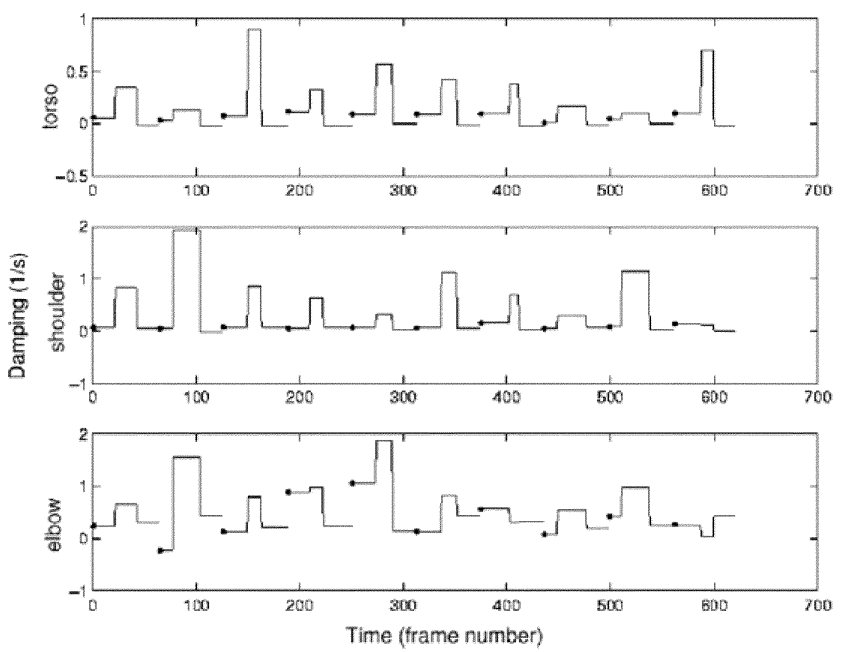

(b)
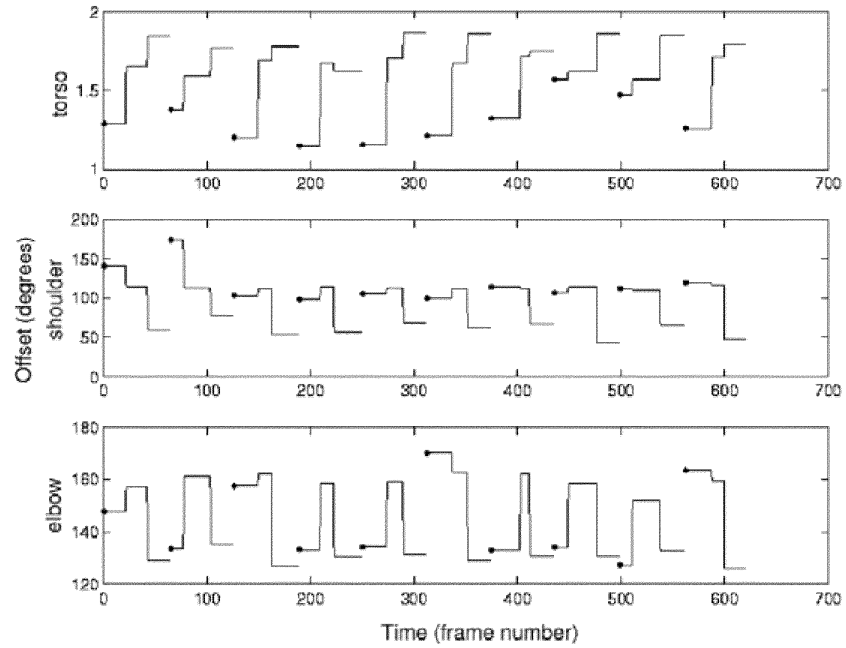

(d)

Fig. 5. These plots graphically show the dynamic model. Ten cycles of the target reaching task are shown with the beginning of each motion cycle is indicated by . Within each cycle, the values computed for the dynamic model parameters on each segment is represented as a step function (which changes step at each segment). The plots show (a) amplitude (degrees). (b) Damping factor (1/s). (c) Frequency (Hz). (d) Offset (degrees) for three components of the motion data, from top to bottom, respectively.

$a_{m n}\left(t_{j}\right)$ is transformed to frequency domain, $A_{m n}\left(f_{k}\right)$ using the fast Fourier transform (FFT), where $f_{k}$ is the discrete frequency. The power spectral density magnitude is given by

$$
A_{m n}^{2}\left(f_{k}\right)=\frac{1}{K^{2}}\left|A_{m n}\left(f_{k}\right)\right|^{2} .
$$

The total power $\bar{A}_{m n}^{2}$ of the element $n$ over $M$ cycles is summed over all frequencies $f_{k}$

$$
\bar{A}_{m n}^{2}=\sum_{k=1}^{K} A_{m n}^{2}\left(f_{k}\right)
$$

By Parseval's theorem for periodic signals, the rms of $a_{m n}\left(t_{j}\right)$ is simply the square root of $\bar{A}_{m n}^{2}$

$$
\mathrm{rms}=\sqrt{\frac{1}{J} \sum_{j=1}^{J} a_{m n}^{2}\left(t_{j}\right)}=\sqrt{\sum_{k=1}^{K} A_{m n}^{2}\left(f_{k}\right)}=\bar{A}_{m n} .
$$

Therefore the average rms, $\bar{A}_{n}$ of the element $n$ is obtained by averaging $\bar{A}_{m n}$ over $M$ cycles and taking the square root

$$
\bar{A}_{n}^{2}=\frac{1}{M} \sum_{m=1}^{M} \bar{A}_{m n}^{2}
$$

resulting in a single quantity that represents motion for each element.

Although the spectral analysis approach permits the averaging of element data and the reduction of the movement data into a single quantity, rms, an undesirable consequence is that frequency information is lost. We will show that by using the kinetic energy instead of rms, frequency is combined with magnitude which results in a single metric that accounts for recognized musculoskeletal exposure factors, including repetitiveness, posture stress and duration.

Assume $x(t)$ is a temporal process with power spectrum $A^{2}(f)$ as a component of the motion $\mathbf{X}(t)$ of a body with mass. 


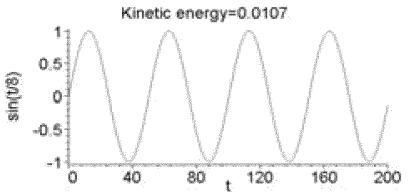

(a)

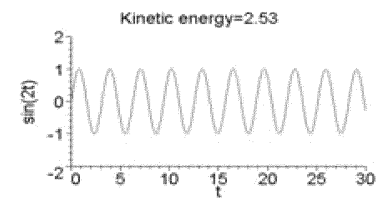

(c)

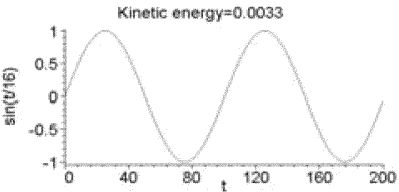

(b)

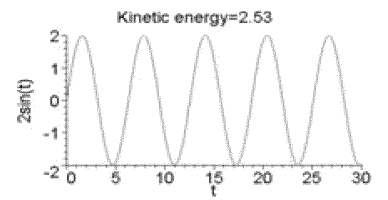

(d)
Fig. 6. (a) and (b) Sine waves in the upper row have the same rms, while the kinetic energy increases for increasing frequency. (c) and (d)The kinetic energies of both sine waves are the same despite their different rms and frequencies, indicating those two waves, as posture data, would result in the equivalent repetitiveness and physical stress if using our kinetic energy measure $\widehat{\mathrm{KE}}$.

The power spectrum of the corresponding velocity $v(t)=\dot{x}(t)$ is calculated by

$$
\begin{aligned}
\dot{A}(f) & =\left|\int_{-\infty}^{\infty} v(t) e^{-j 2 \pi f t} d t\right|^{2} \\
& =\left|\int_{-\infty}^{\infty} x(t) d e^{-j 2 \pi f t}\right|^{2} \\
& =(2 \pi f)^{2}\left|\int_{-\infty}^{\infty} x(t) e^{-j 2 \pi f t} d t\right|^{2} \\
& =(2 \pi)^{2} f^{2} A^{2}(f)
\end{aligned}
$$

which is proportional to $f^{2} A^{2}(f)$. Again by Parseval's theorem, the mean kinetic energy associated with the motion is proportional to $\int_{-\infty}^{\infty} f^{2} A^{2}(f) d f$, which has discrete form

$$
\widehat{\mathrm{KE}}=\dot{A}_{n}=\sqrt{\frac{1}{M} \sum_{m=1}^{M} \sum_{k=1}^{K} f_{k}^{2} A_{m n}^{2}\left(f_{k}\right)}
$$

(and multiplication by the mass yields the true kinetic energy ${ }^{1}$ ). We use the notation $\widehat{\mathrm{KE}}$ to distinguish this measure from the true kinetic energy.

Comparing (11) and (12), one sees that by using $\widehat{\mathrm{KE}}$ as the metric, both frequency and magnitude are explicitly included in the expression of $\dot{A}_{n}$. The kinetic energy-like measure is also consistent with Marras and Schoenmarklin's [16] result that velocity and acceleration data of wrist motion discriminated significant differences between low- and high-risk groups.

An example we compute $\widehat{\mathrm{KE}}$ using synthetic data for the data given in Fig. 6. Two sine waves were used because they are simple and intuitive. On the left of Fig. 6, two sine waves having the same rms are shown in the upper and lower figures. The kinetic energies associated with these time series are $0.0107,0.0033$, respectively. However, the higher frequency (upper figure) yielded a higher value for $\widehat{\mathrm{KE}}$. The right-hand side (RHS) of Fig. 6 shows two sine waves that have different rms and frequencies, but lead to the same value of $\widehat{\mathrm{KE}}(2.53)$,

\footnotetext{
${ }^{1}$ Here, technically the measured quantity is velocity which, as indicated, is proportional to kinetic energy.
}

indicating those two waves, as posture data, would result in the equivalent repetitiveness and physical stress if using $\widehat{\mathrm{KE}}$ as a measure. This simple example illustrates that the single quantity (12) achieves the goal of a frequency-weighted filter. In [22] it was suggested that a frequency-weighted filter would be desirable such that sine waves having the same rms input amplitude should have high-pass filtered rms output amplitudes that increase for increasing frequencies. Furthermore, it was proposed that the high-pass filter cutoff frequency and slope be scaled so equivalent outputs would be obtained for inputs of different combinations of frequency and amplitude (that presumably resulted in equivalent repetitiveness and physical stress).

In a multijoint system the measure $\widehat{\mathrm{KE}}$ computed for each joint can be combined into a single measure if one weights the contribution from each joint based on the relative mass of the joints. Mass distribution tables [18] can be used to obtain estimates for these values, hence a combined metric is possible (e.g., for a male in the 50th percentile for weight, the forearm represents $1.2 \%$, and the upper arm represents $2.1 \%$ of the body mass). The combined metric, however, will be an approximation to the actual kinetic energy because of the estimated mass value.

\section{EXPERIMENTS}

In this section we present an experiment to demonstrate the steps of our algorithm (motion segmentation, motion representation using dynamic model fitting parameters, measurement of kinetic energy). A reaching task is used: reach a target, pause, retract hand to resting position. The task was performed in a laboratory setting, however, it mimics motions performed at a local manufacturing facility (where seated workers picked up an item from a bin at a lower position and placed it on one of three "conveyors" that were roughly at head height, then returned their hand to the lower position). In our laboratory, two subjects of substantially different builds performed this reaching task. The subjects were asked to sit in a chair of fixed height and reach for three targets at varying heights with respect to the chair. The distance from the highest target to the middle one was $11.5 \mathrm{~cm}$ and from the middle to the lowest target was $10.6 \mathrm{~cm}$. The task was repeated with the smaller subject seated in a higher chair (approximately $20 \mathrm{~cm}$ higher than the first chair) while performing the same motion. Each subject was asked to reach for each target three times, followed by a sequence of reaching for each target once (high, medium, low), then in the random order. The pattern was chosen to give sufficient variation to the motion to demonstrate robustness of the segmentation and dynamic model fitting given the variation that occurred while performing the task.

For the task described, joint angle data was measured from our vision-based data acquisition system [14]. Four example images of the smaller subject sitting on the lower chair are shown in Fig. 3(a)-(d). The results of the automated analysis are given in Figs. 4-9. The cycle time of each subject on each motion is approximately the same, 62 frames. The raw motion data for one 

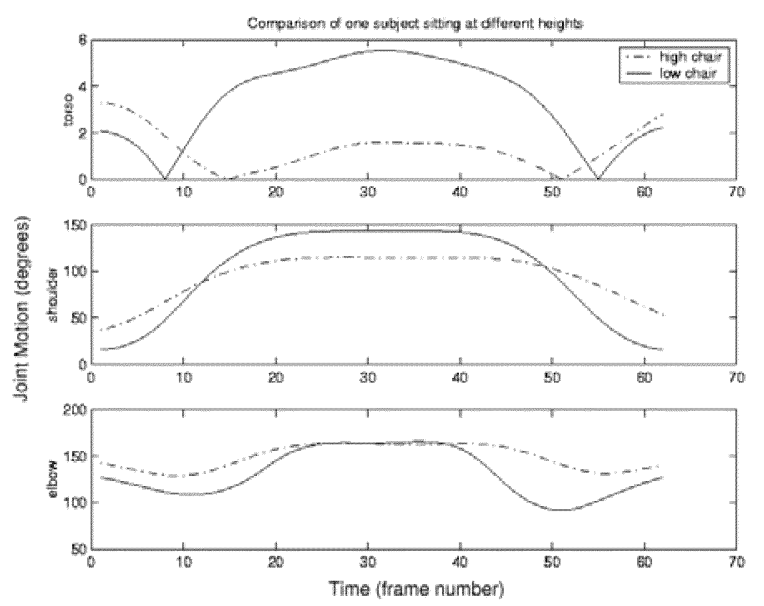

(a)
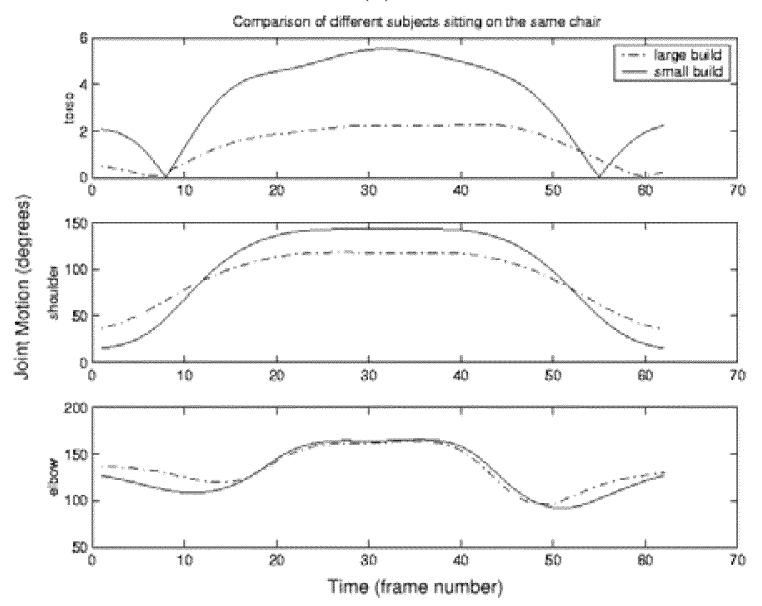

(b)

Fig. 7. Comparison of the motion. (a) One subject seated at two different heights shows much larger motion when seated at on a lower chair. (b) Different subjects when seated on a chair at the same height shows larger motion for the subject with smaller limbs. In each case only one cycle of motion is shown (subjects are reaching the same target).

subject is shown in Fig. 4. Fig. 7(a) shows a single task cycle extracted from the data for one subject using two different height chairs (the smaller subject data is presented here). Fig. 7(b) shows motion of a single cycle of the reaching task for two subjects of different size using a chair of the same height Fig. 8 shows an example of the joint angle data and the computed kinetic energy of the same subject reaching three different targets. $\widehat{\mathrm{KE}}$ was computed for each of the three joint angles studied here (torso, shoulder elbow) as an average over 10 repeated motions of the same reaching task. It is clearly seen that high values of $\widehat{\mathrm{KE}}$ are computed when reaching the higher target than the lower one, as one might expect.

The values computed for $\widehat{\mathrm{KE}}$ for each limb are combined using relative masses for a 50 percentile mass male (larger subject) and 5 percentile mass female subject. The mass ratios for forearm:upper arm: torso (above L5) used were 1.2:2.1:35.8 for the average male and 0.7:1.3:22.4 for the smaller female. The values were obtained from [18]. Fig. 9(a) compares the value of $\widehat{\mathrm{KE}}$ for the same subject using different height chairs (here the data for the smaller subject are presented). For this sub-
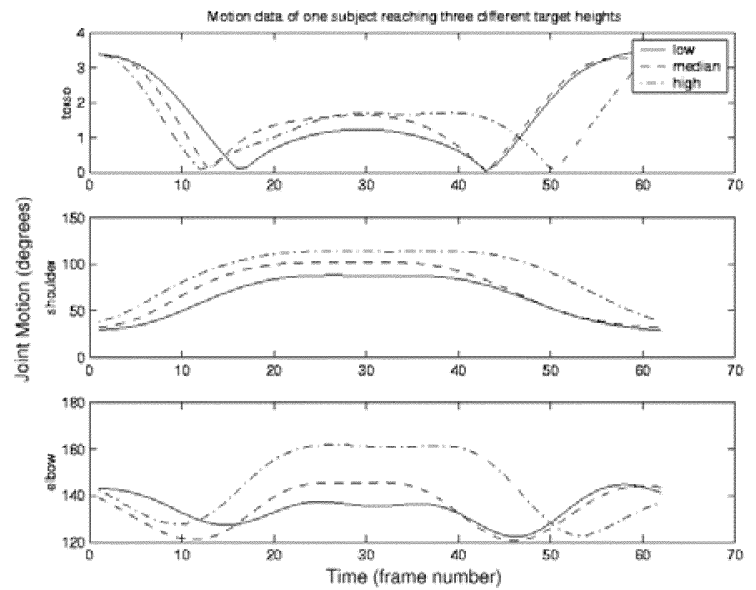

(a)

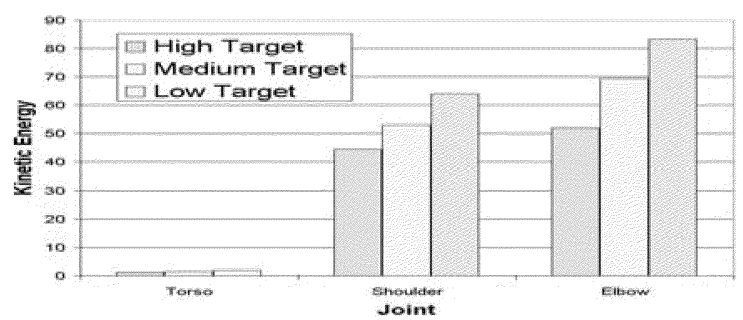

(b)

Fig. 8. A comparison of one subject reaching three different target heights (a) joint angle data. (b) Kinetic energy for each of the three joints. The kinetic energy is computed as the average of 10 motions (or the same reaching task).

ject values of $\widehat{\mathrm{KE}}$ are smaller when seated in the higher chair because the subject did not need to move as far to reach the target. Fig. 9(b) compares different size subjects using the same chair. Larger values of $\widehat{\mathrm{KE}}$ were measured for the smaller subject reaching the targets.

When comparing $\widehat{\mathrm{KE}}$ computed for the subject with smaller limb size seated at the higher chair position with $\widehat{\mathrm{KE}}$ for the larger subject seated on the lower chair, the values for all three joints are very close [see Fig. 9(c)] indicating that when using $\widehat{\mathrm{KE}}$ as the metric, the repetitiveness and posture stress of both subjects is roughly equivalent if the seating position is adjusted to accommodate the limb size differences. This result makes sense because the distance the subject has to reach, relative to their limb size, can be made approximately the same by adjusting the chair height-thus their motion (and kinetic energy) should be similar.

\section{SUMMARY AND DISCUSSION}

Systems that measure human motion are applicable in a wide variety of applications and there is huge potential for telemonitoring of patients or occupational risk. When represented as a time series, the high dimensionality and small variations in time make it difficult to make assessments or comparisons between human movements. Our solution is to represent the motion as a concatenation of simple motions (damped harmonic motion) and use the parameters of the dynamic system to compactly represent motion. This compact representation is suitable for nu- 


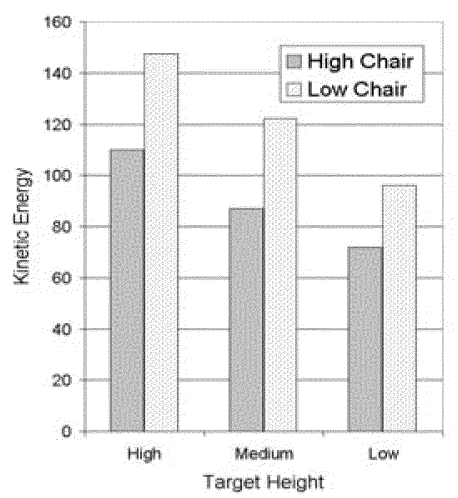

(a)

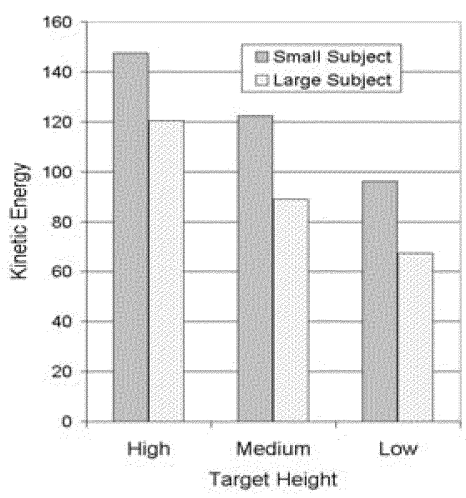

(b)

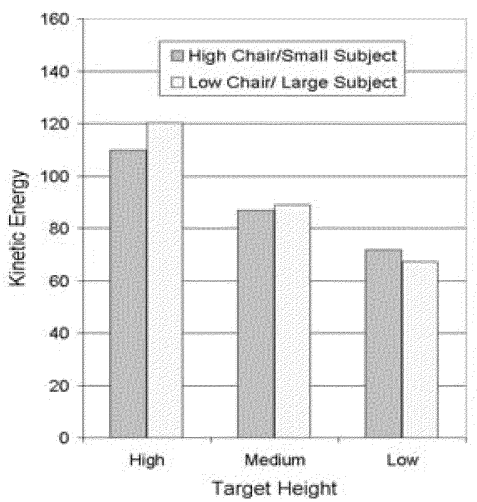

(c)

Fig. 9. Comparison of kinetic energy measure, $\widehat{\mathrm{KE}}$ with (a) different heights of chairs or (b) different subjects. It is shown clearly from the graphs that higher values of $\widehat{\mathrm{KE}}$ are measured when sitting in the lower chair. or when a subject with smaller limb size is performing the task. (c) When sitting at a higher chair, the measured $\widehat{\mathrm{KE}}$ of the smaller subject is similar to that of the larger subject sitting at the lower chair, indicating the repetitiveness and posture stress of the second subject is equivalent with the first subject only when seated at a higher position due to the relatively small limb size of the second subject.

merous applications such as telemedicine and ergonomics. Success of such a representation relies on the ability to decompose the motion data into such segments, and we have presented an algorithm to achieve such a decomposition. We have presented an algorithm that takes as input a multidimensional time series of joint motion data and decomposes the data into segments such that a second-order AR model adequately describes the motion on each segment. Four parameters of the dynamic model were used to describe the motion on each segment. These parameters, along with the epoch (time) for each segment form a compact representation of the motion, i.e., the time series data can be reduced to a set of $5 \times s$ parameters. This representation yields motion statistics, such as frequency information, that are currently utilized in ergonomic studies as well as enabling other metrics to be developed. A kinetic energy-like measure, $\widehat{\mathrm{KE}}$, computed using the parameters of the dynamic model, has been proposed as a suitable comparison for motion repetition. This measure includes both the rms and frequency aspects of the motion. Experiments demonstrated that this representation is robust to small variations in the motion (both in the temporal and spatial aspects of the motion). Experiments presented on upper-body motion demonstrated the ability to handle multijoint motion. The kinetic energy measure is computed for each joint and combined using estimates for the relative mass of the limbs.

Assessing work-place risk is of critical importance and the introduction of a metric for comparing human motion requires significant study before claims can be made about its utility in assessing posture stress. Automated analysis of motion data will be crucial to provide robust, repeatable information. We have introduced one candidate $\widehat{\mathrm{KE}}$ that yields a metric useful for comparing task motion across difference subject sizes. Further experimentation with ergonomic practitioners is underway to evaluate the applicability of this measure.

\section{REFERENCES}

[1] 2000: TLV's and BEIs: Threshold Limit Values and Biological Exposure Indices. Cincinnati, OH: ACGIH Worldwide, 2000, pp. 117-121.

[2] P. Andersson, "Adaptive forgetting in recursive identification through multiple models," in Proc. Int. J. Contr., 1985, pp. 1175-1193.
[3] T. J. Armstrong, D. B. Chaffin, and J. A. Foulke, "A methodology for documenting hand positions and forces during manual work," $J$. Biomechan., vol. 12, pp. 131-133, 1979.

[4] T. J. Armstrong, J. A. Foulke, B. Joseph, and S. Goldstein, "An investigation of cumulative trauma disorders in a poultry processing plant," Amer. Industrial Hygiene Assoc. J., vol. 43, pp. 103-116, Apr. 1982.

[5] T. J. Armstrong, R. G. Radwin, D. J. Hensen, and K. W. Kennedy, "Repetitive trauma disorders: Evaluation and design," Human Factors, vol. 28, no. 3, pp. 325-336, 1986.

[6] T. J. Armstrong and B. A. Silverstein, "Upper-extremity pain in the workspace: Role of usage in causality," in Clinical Concepts in Regional Musculoskeletal Illness. New York: Grune \& Stratton, 1987, pp. 333-354.

[7] A. Blake and M. Isard, Active Contours. New York: Springer-Verlag, 1998.

[8] E. N. Corlett, S. J. Madeley, and I. Manenica, "A technique for recording working postures," Ergonomics, vol. 22, no. 3, pp. 357-366, 1979.

[9] M. Gleicher, "Animation from observation: Motion capture and motion editing," Computer Graphics, vol. 33, no. 4, Nov. 1999.

[10] I. Karatzas and S. E. Shreve, Brownian Motion and Stochastic Calculus: Springer, 1991.

[11] W. M. Keyserling, "A computer-aided system to evaluate posture stress in the workplace," Amer. Industrial Hygiene Assoc. J., vol. 47, pp. 641-649, 1986.

[12] H. Liu, "Segmenting and Identifying Dynamic Models from Measurement of Visual Motion," Master's Thesis, Dept. Mech. Eng., Univ. Wisconsin-Madison, 1999.

[13] C. M. Lu and N. J. Ferrier, "Segmentation and event classification for the analysis of repetitive motion," IEEE Trans. Pattern Anal. Machine Intell., vol. 26, Feb. 2004, to be published.

[14] C. M. Lu and N. J. Ferrier. (2001) A system for the Automated Measurement of Human Motion During Repetitive Tasks [Online]. Available: http://robios8.me.wisc.edu/publications.html

[15] C. M. Lu, H. Z. Liu, and N. J. Ferrier, "Multidimensional motion segmentation and identification," Proc. IEEE Conf. Computer Vision and Pattern Recog., pp. 629-636, 2000.

[16] W. S. Marras and R. W. Schoenmarklin, "Wrist motions industrial," $E r$ gonomics, vol. 36, no. 4, pp. 341-351, 1993.

[17] A. Menache, Understanding Motion Capture for Computer Animation and Video Games. New York: Morgan Kaufman (Academic), 2000.

[18] "Survey, Weight, Height, and Selected Body Dimensions of Adults," National Health Survey, ser. 11, PHS Publ. 1000, 1965.

[19] P. Kansi, O. Karhu, and I. Kuorinka, "Correcting working postures in industrial: A practical method for analysis," Appl. Ergonom., vol. 8, pp. 199-201, 1977.

[20] V. Z. Priehl, "A numerical definition of posture," Human Factors, vol. 16, no. 6, pp. 576-584, 1974.

[21] R. G. Radwin and M. L. Lin, "An analytical method for characterizing repetitive motion and posture stress using spectral analysis," Ergonomics, vol. 36, no. 4, pp. 379-389, 1993.

[22] R. G. Radwin, M. L. Lin, and T. Y. Yen, "Exposure assessment of biomechanical stress in repetitive manual work using frequency-weighted filters," Ergonomics, vol. 37, no. 12, pp. 1984-1998, 1994. 
[23] B. A. Silverstein, L. J. Fine, and T. J. Armstrong, "Hand wrist cumulative trauma in industry," Brit. J. Indust. Med., vol. 43, pp. 779-984, 1986.

[24] R. Wells, A. Moore, and J. Cholewicki, Evaluation of Upper Limb Stresses Using Musculoskeletal Loads During a Rotating Light Assembly Task: Taylor \& Francis, 1990.

[25] T. Y. Yen and R. G. Radwin, "Comparison between using spectral analysis of electrogoniometer data and observational analysis to quantify repetitive motion and ergonomic changes in cyclic industrial work," Ergonomics, vol. 43, no. 1, pp. 106-132, 2000.

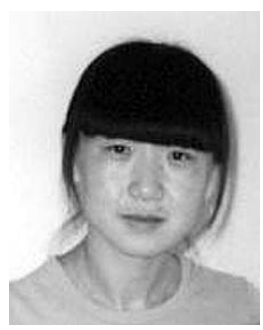

ChunMei Lu received the B.S., M.S., and Ph.D. degrees in computational mathematics at Jilin University, P.R. China, in 1990, 1993, and 1997, respectively, and the M.S. degree in computer sciences from the University of Wisconsin-Madison in 2001.

She is a Ph.D. candidate in the Mathematics and Computation in Engineering (MaCE) Program at the University of Wisconsin-Madison. Her current research focus is on complex motion analysis: learning dynamics to track complex motion and developing metrics to compare motions.

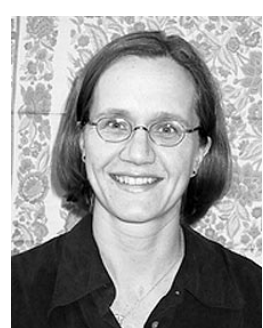

and dynamic tracking.
Nicola J. Ferrier (S'90-M'92) received the B.Sc. degree in applied mathematics and the M.Sc. degree in computing sciences from the University of Alberta, Edmonton, Alberta, Canada, and the S.M. and Ph.D. degrees from the Division of Engineering and Applied Sciences, Harvard University, Cambridge, MA.

She is an Associate Professor with the Department of Mechanical Engineering, University of WisconsinMadison. Her research focuses on the integration of visual sensors in robot control systems, concentrating primarily on active vision systems, attentive control, 